\title{
State-space models for optical imaging
}

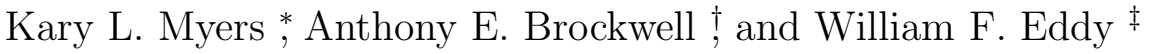

August 14, 2006

\begin{abstract}
Measurement of stimulus-induced changes in activity in the brain is critical to the advancement of neuroscience. Scientists use a range of methods, including electrode implantation, surface (scalp) electrode placement, and optical imaging, to gather data capturing underlying signals of interest in the brain. These data are usually corrupted by artifacts, complicating interpretation of the signal; in the context of optical imaging, two primary sources of corruption are the heartbeat and respiration cycles. We introduce a new linear state-space framework which uses the Kalman filter to remove these artifacts from optical imaging data. The method relies on a likelihood-based analysis under the specification of a formal statistical model, and allows for corrections to the signal based on auxiliary measurements of quantities closely related to the sources of contamination, such as physiological processes. Furthermore, the likelihood-based modeling framework allows us to perform goodness-of-fit testing and to perform formal hypothesis testing on parameters of interest. Working with data collected by our collaborators, we demonstrate the method on data collected in an optical imaging study of a cat's brain.
\end{abstract}

KEY WORDS: linear state-space models, Kalman filtering, functional neuroimaging, optical imaging, orientation columns.

\section{Introduction}

Hemodynamic-metabolic methods of functional neuroimaging, such as functional magnetic resonance imaging (fMRI) (Ogawa et al., 1992) and optical imaging (Grinvald et al., 1986), rely on the hypothesis that changes in brain activity lead to local changes in metabolic demands, which in turn lead to changes in blood volume and flow (Grinvald et al., 2000). Working backward, scientists can measure localized changes in metabolic activity and then infer changes in neural activity. Both fMRI and optical imaging are thought to measure changes in blood volume, blood flow, and blood oxygen levels in response to neural activity. In an experimental setting, one typically measures this neural activity, which we will refer to as activation, and then performs analysis of the connection between activation and various stimuli. Such analysis has been used, for instance, to build maps of so-called orientation columns in the visual cortex (Hübener and Bonhoeffer, 2002).

Optical imaging has the advantage of having significantly higher spatial resolution than most other methods of in-vivo functional neuroimaging and higher temporal resolution than many (Grinvald et al., 1999). Thus it has the potential to be a powerful tool for mapping the brain's functional architecture.

\footnotetext{
* Statistical Sciences Group, Los Alamos National Laboratory, Los Alamos, NM 87545

$\dagger$ Department of Statistics, Carnegie Mellon University, Pittsburgh, PA 15213

${ }^{\ddagger}$ Department of Statistics, Carnegie Mellon University, Pittsburgh, PA 15213
} 
To collect optical imaging data, a scientist shines visible light on the exposed surface of the brain and measures reflected light with a digital video camera. Neural activity changes the intensity of the reflected light captured by the camera, making it possible to measure differences between active and inactive brain tissue. Unfortunately, optical imaging typically suffers from very low signal-to-noise ratios which mask these measurable differences. The images also include variation unrelated to the activation, especially from physiological sources like heartbeat and respiration; often this unrelated variation is of greater magnitude than the variation due to the activation.

A standard way to deal with this problem is simply to take the average of all video frames recorded during one stimulus and subtract from this the average of all the video frames recorded during a complementary stimulus. This standard difference method tries to remove unrelated variation by averaging data. Several researchers (e.g., Stetter et al., 2000; Gabbay et al., 2000; Schießl et al., 1999) have improved on this approach by first factoring the data, as with principal component analysis or independent component analysis, before applying the standard difference procedure.

In this paper we propose a new method based on linear state-space models (sometimes called linear dynamical systems) to reveal brain activation in optical imaging data, taking into account possible external physiological measurements. The approach provides a flexible framework for estimating activation in each pixel of the video data using an efficient algorithm called the Kalman filter, and it has several advantages over existing methods. It provides a more systematic way of accounting for artifacts induced, for instance, by heartbeat and respiration. Since the approach is likelihood-based and yields conditional distributions of activation levels, given observations, it can be used to construct formal confidence intervals for activation as well as for model parameters of interest. Furthermore, since the Kalman filter is recursive (in time), it can be used within our framework to carry out real-time activation estimation.

The remainder of this paper is organized as follows. We describe optical imaging in more detail in Section 2 and introduce the video data and auxiliary measurements we will be considering. We review basics of the linear state-space model framework and the Kalman filter in Section 3. We then describe our general image analysis procedure in Section 4, and build and analyze a model for our data, showing how our results are consistent with widely accepted principles of orientation-tuning for neurons in the visual cortex. Finally we discuss various refinements of the scheme in Section 5.

\section{Optical Imaging}

\subsection{Background on Optical Imaging and Orientation Columns}

As described briefly above, optical imaging involves using a digital video camera called a charge-coupled device (CCD) camera to record visible light (often 570 or 620 nanometers) that is reflected off the surface of the brain. Scientists typically use optical imaging to study laboratory animals rather than humans, although a human undergoing neurosurgery can be a subject for an optical imaging study. For each pixel, the camera records a value proportional to the number of photons hitting that pixel location on the camera's detector. Brain activity changes the intensity of the reflected light and hence the count of photons at each pixel location.

Many neurons in the primary visual cortex are "tuned" to respond to the orientation- e.g., horizontal, vertical - of the visual stimulus (Hübener and Bonhoeffer, 2002). Neurons tuned to the same orientation form column-like structures extending through the layers of the cortex (Hubel and Wiesel, 1963). In a 


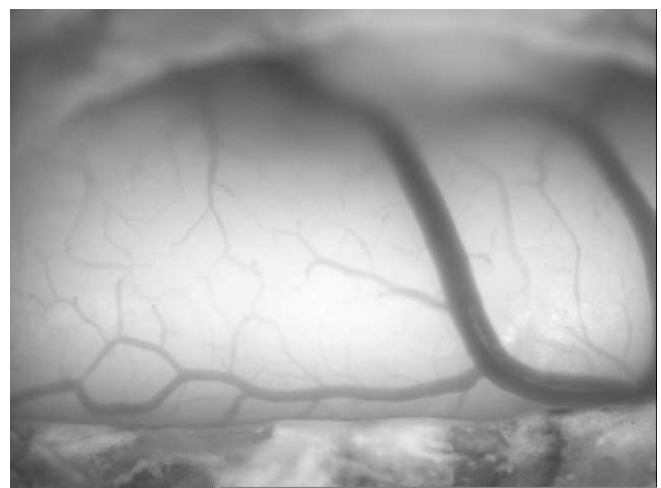

Figure 1: One video frame, $640 \times 480$ pixels, of a 40 square millimeter area on the surface of a brain. Some features of interest: the vascular artifacts seen as dark branching structures; the edges of the incised dura, a membrane surrounding the brain, visible near the top and bottom of the image; and the uneven lighting due to the curved surface of the cortex and the uneven distribution of light.

typical experiment to stimulate these orientation columns, the scientist uses a CRT monitor to present moving black-and-white gratings in each of the orientations of interest, perhaps interspersed with a blank gray screen.

Scientists have understood how to elicit activation in orientation columns for decades; in themselves orientation columns are no longer of real scientific interest. However, these experiments make a good test bed for developing new analysis techniques precisely because they are so well understood. In particular, in our data analysis, we will examine results with respect to their consistency with known behaviour of orientation columns.

\subsection{Data}

To illustrate and assess the performance of our proposed methodology, we will use data collected by SeongGi Kim's research group at the University of Pittsburgh's Magnetic Resonance Research Center in the Department of Radiology. The lab collects video frames like the one in Figure 1 at a rate of 30 frames per second, with each video frame containing $640 \times 480=307,200$ pixels. Each pixel is $11 \times 13$ microns, which is about the size of a very small cell.

We consider an experiment in which the scientist presented visual stimuli in four orientations: 0 degrees, 45 degrees, 90 degrees, and 135 degrees. Each orientation is presented for two seconds and followed by two seconds of a blank gray screen, yielding 120 video frames per orientation at a rate of 30 frames per second. One cycle through each orientation-plus-gray combination equals one trial, so there are 120 frames $\times 4$ orientations $=480$ frames per trial. The scientist performed 20 trials, for $T=9,600$ total frames of video data in this experiment.

At the same time that the Kim Lab records video of the surface of the brain, they also record several channels of physiological measurements, including blood pressure (to assess vascular changes), EEG (to measure overall brain activity), and expired carbon dioxide (to track respiration and as a proxy for metabolism). This simultaneous recording of physiological channels is not standard optical imaging laboratory practice, and it offers us the opportunity to correct the images for physiological variation directly rather than using 


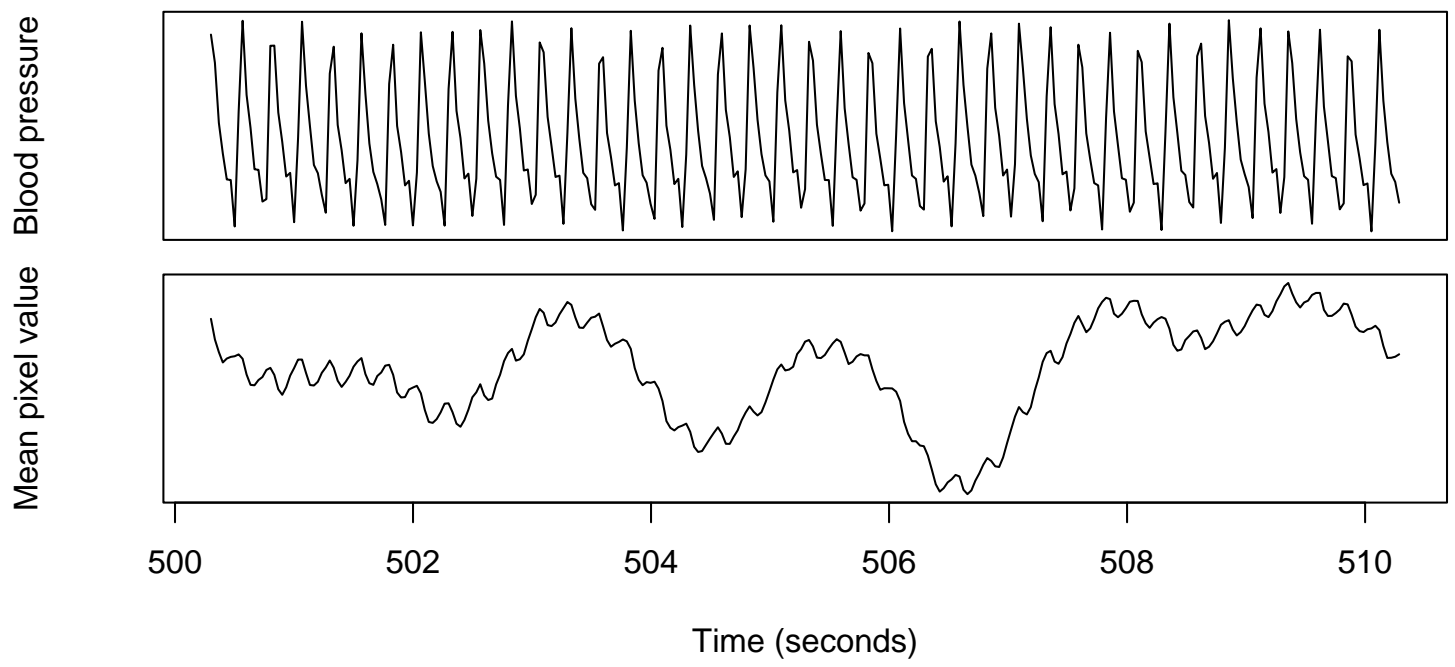

Figure 2: A ten-second subset of the time series of blood pressure readings (top) and the mean pixel value (bottom). Note the "pulse" - an oscillation which repeats about 40 times during these ten seconds - which can be seen in both time series. This corresponds to the heartbeat with a rate of about 240 beats per minute in this experiment. The two time series also show a slower oscillation, repeating about five times in this subset, corresponding to the respiration of 29 breaths per minute.

averaging to smooth it out or factoring to separate it out. In this paper we focus on the blood pressure channel and how it relates to the images of the surface of the brain.

A little preliminary analysis serves to highlight the importance of the auxiliary measurements. Consider the two time series in Figure 2, collected during a typical optical imaging experiment. The top plot shows blood pressure readings over a ten-second time interval. The obvious cyclic component corresponds to the heartbeat, with a rate of around 240 beats per minute. The bottom plot shows the mean pixel intensity (averaged over all 307,200 image pixels) during the same ten seconds. The pulse that is the main feature in the blood pressure plot can also be seen as a smaller-magnitude oscillation in the mean pixel data. In addition, the mean pixel data show a slower oscillation caused by the respiration of around 29 breaths per minute. While not as easy to see in the plot of blood pressure, explorations in (Myers, 2004) demonstrate that the variation due to respiration is also present there.

To establish the connection more formally, we can evaluate sample cross-correlations between the blood pressure and the mean pixel intensity time series. These cross-correlations are shown in Figure 3. The figure shows how the correlation depends on both the heartbeat cycle and the respiration cycle.

\section{$3 \quad$ State-Space Model Preliminaries}

Before describing how our method works, we need to give (standard) definitions of linear state-space models and review the Kalman filtering recursions. 


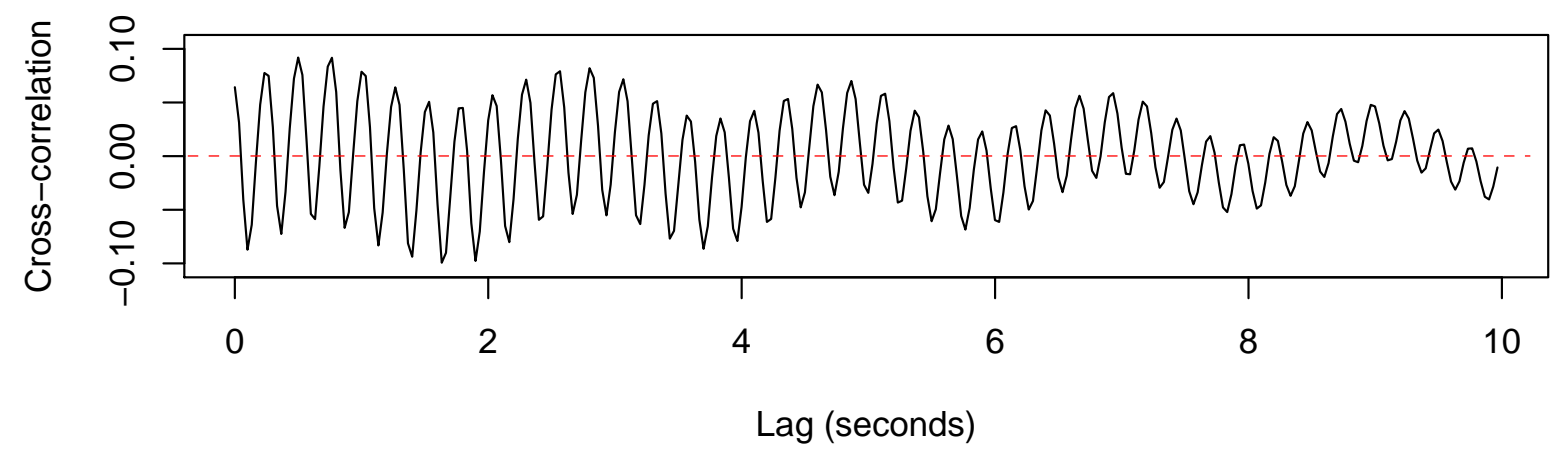

Figure 3: Sample cross-correlation of blood pressure with the mean pixel value for the first 300 lags (ten seconds). Each point indicates the correlation between blood pressure and pixel value at that lag. Note the low and high frequency oscillations corresponding to respiration and heartbeat, respectively.

\subsection{Formal Definition of a Linear Gaussian State-Space Model}

A linear Gaussian state-space model (described more fully in (Brockwell and Davis, 1991)) is specified by the state equation

$$
\underbrace{\boldsymbol{X}_{t+1}}_{m \times 1}=\underbrace{F_{t}}_{m \times m} \underbrace{\boldsymbol{X}_{t}}_{m \times 1}+\underbrace{\boldsymbol{V}_{t}}_{m \times 1}, \quad t=1,2, \ldots, T
$$

and the observation equation

$$
\underbrace{\boldsymbol{Y}_{t}}_{n \times 1}=\underbrace{G_{t}}_{n \times m} \underbrace{\boldsymbol{X}_{t}}_{m \times 1}+\underbrace{\boldsymbol{W}_{t}}_{n \times 1}, \quad t=1,2, \ldots, T
$$

where $\left\{F_{1}, F_{2}, \ldots\right\}$ is a sequence of $m \times m$ matrices called state transition matrices, $\left\{G_{1}, G_{2}, \ldots\right\}$ is a sequence of $n \times m$ matrices called observation matrices, $\left\{\boldsymbol{V}_{t}\right\}$ is an independent and identically distributed (iid) sequence of $N(0, Q)$ random vectors, and $\left\{\boldsymbol{W}_{t}\right\}$ is an iid sequence of $N(0, R)$ random vectors. To complete the specification of the model, we also assume that $\boldsymbol{X}_{1} \sim \mathrm{N}\left(\boldsymbol{\mu}_{1}, \Sigma_{1}\right)$.

The state sequence $\left\{\boldsymbol{X}_{t}\right\}$ is assumed to be unobservable. The observation sequence $\left\{\boldsymbol{Y}_{t}\right\}$, on the other hand, can be observed. A typical goal of analysis is to make inferences at each time $t$ about the unobserved state $\boldsymbol{X}_{t}$ given observations $\boldsymbol{Y}_{1}, \ldots, \boldsymbol{Y}_{t}$. In our context, we will construct a model for each pixel $s$. As described in Section 4.2, our state vector $\boldsymbol{X}_{t}$ will include (among other things) the activation signal $a_{t}$ that we are interested in, while our observation "vector" $\boldsymbol{Y}_{t}=y_{t}$ will contain only the measured video signal, a scalar value at each pixel.

Note that we could include a subscript $s$ in the model specification to indicate which pixel is being considered. For simplicity, we suppress this subscript throughout this paper, but it should be kept in mind that in general the computations described here need to be performed for each pixel. 


\subsection{The Kalman Filter}

To use the model described by (1) and (2) for activation level estimation, we need to be able to perform two tasks. The first is to find the conditional expectations and covariances

$$
\hat{\boldsymbol{X}}_{t \mid u}=\mathrm{E}\left(\boldsymbol{X}_{t} \mid y_{1}, \ldots, y_{u}\right), 1 \leq u \leq t
$$

and

$$
\Sigma_{t \mid u}=\operatorname{Cov}\left(\boldsymbol{X}_{t} \mid y_{1}, \ldots, y_{u}\right), 1 \leq u \leq t .
$$

In particular, we are interested in finding these when $u=t$, in which case the problem is referred to as the filtering problem, and when $u=t-1$, in which case the problem is called the prediction problem.

The second task is to fit appropriate models to our data. In other words, we need to find appropriate matrices $F_{t}, G_{t}, Q$, and $R$. It turns out that solving the prediction problem will enable us to perform this task (as described below).

The first task has a direct consequence in terms of estimation of the state vectors $\boldsymbol{X}_{t}$. In particular, for each $t, \hat{\boldsymbol{X}}_{t \mid t}$ is the point estimate of $\boldsymbol{X}_{t}$ that minimizes mean-squared error, given observations $y_{1}, \ldots, y_{t}$. Since we construct our state vector so as to include the unknown activation signal $a_{t}$, this provides us with a formal way to obtain minimum mean-squared error estimates of our activation signal at each point in time.

\subsubsection{Filtering and Prediction}

The Kalman filter (Kalman, 1960) is a recursive scheme for obtaining the conditional expectations and covariances $\hat{\boldsymbol{X}}_{t \mid t}, \hat{\boldsymbol{X}}_{t \mid t-1}, \Sigma_{t \mid t}$, and $\Sigma_{t \mid t-1}$. It is recursive in the sense that if we are given these quantities for some value of $t$, then we only need the value of the observation $y_{t+1}$ in order to compute $\hat{\boldsymbol{X}}_{t+1 \mid t+1}, \hat{\boldsymbol{X}}_{t+1 \mid t}$, $\Sigma_{t+1 \mid t+1}$, and $\Sigma_{t+1 \mid t}$. We initialize the procedure with $\hat{\boldsymbol{X}}_{1 \mid 0}=\boldsymbol{\mu}_{1}$ and $\Sigma_{1 \mid 1}=\Sigma_{1}$ (recall that these quantities are specified as part of the state-space model). The Kalman recursions are

$$
\begin{aligned}
\hat{\boldsymbol{X}}_{t+1 \mid t+1} & =\hat{\boldsymbol{X}}_{t+1 \mid t}+K_{t+1}\left(y_{t+1}-G_{t+1} \hat{\boldsymbol{X}}_{t+1 \mid t}\right) \\
\Sigma_{t+1 \mid t+1} & =\left(I-K_{t+1} G_{t+1}\right) \Sigma_{t+1 \mid t},
\end{aligned}
$$

where

$$
\begin{aligned}
\hat{\boldsymbol{X}}_{t+1 \mid t} & =F_{t} \hat{\boldsymbol{X}}_{t \mid t}, \\
\Sigma_{t+1 \mid t} & =F_{t} \Sigma_{t \mid t} F_{t}^{T}+Q,
\end{aligned}
$$

and the so-called Kalman gain term $K_{t+1}$ is given by

$$
K_{t+1}=\Sigma_{t+1 \mid t} G_{t+1}^{T}\left[G_{t+1} \Sigma_{t+1 \mid t} G_{t+1}^{T}+\sigma_{y}^{2}\right]^{(-1 *)} .
$$

Here $[\cdot]^{\left(-1^{*}\right)}$ denotes any pseudo-inverse of its argument. (In the optical imaging model we construct in the next section, the argument to the pseudo-inverse is always scalar-valued, making it trivial to calculate.)

We use the conditional mean $\hat{\boldsymbol{X}}_{t \mid t}$ as our estimate of the hidden state $\boldsymbol{X}_{t}$. In our models, the activation level $a_{t}$ will be included in this vector, thus we can extract the conditional mean of the activation signal at time $t$. This point estimate minimizes the mean-squared estimation error for activation under the assumptions that the data are indeed generated by the specified model. 


\subsubsection{Parameter Estimation}

In practice, the matrices $F_{t}, G_{t}, Q$, and $R$ contain parameters which are not known a priori. Maximum likelihood parameter estimation is straightforward to perform using the filtering recursions introduced in the previous subsection. Specifically, let $\boldsymbol{\Theta}$ denote a vector of unknown parameters. Let us also make explicit the dependence of the matrices on $\boldsymbol{\Theta}$ by writing them as $F_{t}(\boldsymbol{\Theta}), G_{t}(\boldsymbol{\Theta}), Q(\boldsymbol{\Theta})$, and $R(\boldsymbol{\Theta})$. Then the likelihood of observed data $\boldsymbol{Y}_{1}, \ldots \boldsymbol{Y}_{T}=y_{1}, \ldots, y_{T}$ as a function of $\boldsymbol{\Theta}$ takes the simple form

$$
L\left(\boldsymbol{\Theta}, y_{1}, \ldots, y_{T}\right)=\prod_{t=1}^{T} \phi\left(y_{t} ; G_{t}(\boldsymbol{\Theta}) \hat{\boldsymbol{X}}_{t \mid t-1}, G_{t}(\boldsymbol{\Theta}) \Sigma_{t \mid t-1} G_{t}(\boldsymbol{\Theta})^{T}+R(\boldsymbol{\Theta})\right),
$$

where the vectors $\hat{\boldsymbol{X}}_{t \mid t-1}$ and matrices $\Sigma_{t \mid t-1}$, for $t=1, \ldots, T$ are obtained by using the recursions in (5), (6), (7), and (8), along with the appropriate matrices $F_{t}(\boldsymbol{\Theta}), G_{t}(\boldsymbol{\Theta}), Q(\boldsymbol{\Theta})$, and $R(\boldsymbol{\Theta})$; and $\phi(y ; \mu, \Sigma)$ denotes the density of a (possibly multivariate) Gaussian random variable with mean $\mu$ and variance $\Sigma$.

Thus the maximum likelihood estimate of the parameters is easily found by embedding the likelihood calculation (10) into a numerical optimization algorithm to find

$$
\hat{\boldsymbol{\Theta}}=\arg \max _{\boldsymbol{\Theta}} \log L\left(\Theta, y_{1}, \ldots, y_{T}\right) .
$$

(Note that to prevent numerical errors, it is usually necessary to maximize the log-likelihood rather than the likelihood itself.)

\section{Analysis Procedure and Application to Imaging Data}

\subsection{General Analysis Procedure}

We propose the following procedure, to be applied for each pixel $s$.

1. Specify the model structure. As laid out in (15)-(17) below, we build the activation levels $a_{t}$ into the hidden state $\boldsymbol{X}_{t}$; we incorporate information about the experimental stimulus in the state transition matrix $F_{t}$; and we include auxiliary physiological measurements in the model by way of the observation matrix $G_{t}$.

2. Use maximum likelihood estimation to determine the model parameters.

3. Recursively compute the quantities

$$
\hat{\boldsymbol{X}}_{1 \mid 1}, \hat{\boldsymbol{X}}_{2 \mid 2}, \ldots, \hat{\boldsymbol{X}}_{T \mid T}
$$

These will serve as our best (in the mean-squared error sense) estimates of the hidden state. The components of these vectors corresponding to the activation levels $a_{t}$ provide minimum mean-squared error estimates of the activation levels.

\subsection{Application to Our Data}

To better illustrate how our method works, we now construct a model for a single pixel of the video data introduced in Section 2.2. First let us define

$$
a_{t}=\text { activation level at time } t
$$




$$
\begin{aligned}
\text { stimulus }_{t} & =\sum_{i \in 0, \ldots, 4} \gamma_{i} p_{t i}, \\
\text { physiology }_{t} & =\beta_{1} b_{t}+\beta_{2} b_{t-1}+\beta_{3} b_{t-2},
\end{aligned}
$$

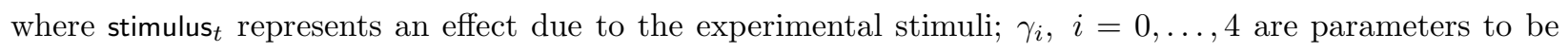
estimated; $p_{t i}$ is an indicator, equal to 1 if experimental stimulus $i$ was presented at time $t$ and 0 otherwise (recall from Section 2.2 that the scientist uses four different stimuli along with a blank gray screen, denoted by $i=0$, in our data); physiology ${ }_{t}$ represents a (moving average) filtered version of the measurements of blood pressure $b_{t}$ which contaminates our signal; and $\beta_{1}, \beta_{2}$, and $\beta_{3}$ are also parameters to be estimated. We are primarily interested in estimating the activation signal $\left\{a_{t}, t=1, \ldots, T\right\}$, which is not directly observable.

Our formal model is given by equations (1) and (2), with

$$
\begin{aligned}
& \boldsymbol{X}_{t}=\left[\begin{array}{c}
a_{t} \\
1
\end{array}\right], \\
& F_{t}=\left[\begin{array}{cc}
\alpha & \text { stimulus }_{t} \\
0 & 1
\end{array}\right], \quad Q=\left[\begin{array}{cc}
\sigma_{a}^{2} & 0 \\
0 & 0
\end{array}\right], \\
& G_{t}=\left[\begin{array}{ll}
1 & \text { physiology }_{t}
\end{array}\right], \quad R=\sigma_{y}^{2},
\end{aligned}
$$

where $\sigma_{a}^{2}$ and $\sigma_{y}^{2}$ are variance parameters, also to be estimated.

Intuitively, the model can be thought of as follows. The activation level follows an autoregressive process driven by an input which depends on the stimulus, as well as some Gaussian noise; that is, $a_{t+1}=\alpha a_{t}+$ stimulus $_{t}+v_{t}$. An autoregressive process makes sense in this context since it has some persistence as we would expect for physical responses in tissue. The driving input levels corresponding to different stimuli are specified by the $\gamma_{i}$ terms in (13), and are not known in advance, but rather are estimated from data.

This activation level is not observed directly, and is contaminated by the filtered version of the blood pressure measurements given in (14). We choose to use a filtered version here (instead of just using an instantaneous effect) since blood pressure measurements are made at the animal's foreleg rather than at the brain. The final model for our observed data $y_{t}$ consists of the activation signal itself, plus the filtered blood pressure signal, along with additional Gaussian noise; i.e., $y_{t}=a_{t}+$ physiology $_{t}+w_{t}$.

This model has eleven parameters: $\alpha$, an autoregressive parameter in the model for the activation $a_{t} ; \sigma_{a}^{2}$, the variance of the state noise $v_{t} ; \sigma_{y}^{2}$, the variance of the observation noise $w_{t} ; \gamma_{i}, i \in 0, \ldots, 4$, which govern the contribution of each orientation of the presented experimental stimuli and the blank gray screen in the stimulus $_{t}$ terms; and $\beta_{j}, j \in 1,2,3$, which measure the contribution of the three lags of blood pressure in the physiology $y_{t}$ terms.

We first find maximum likelihood estimates of the eleven parameters by numerical maximization of (10) under the model specified above. For the pixel we consider, our resulting parameter estimates are $\hat{\alpha}=0.830$; $\hat{\sigma}_{a}^{2}=1.057^{2} ; \hat{\sigma}_{y}^{2}=2.087^{2} ; \hat{\gamma}_{0}=20.559 ; \hat{\gamma}_{1}=20.65 ; \hat{\gamma}_{2}=20.531 ; \hat{\gamma}_{3}=20.489 ; \hat{\gamma}_{4}=20.607 ; \hat{\beta}_{1}=1.92 \times 10^{-5}$; $\hat{\beta}_{2}=-2.77 \times 10^{-6} ;$ and $\hat{\beta}_{3}=2.32 \times 10^{-6}$.

Once this has been done, we simply apply the Kalman recursions to determine $\left\{\hat{\boldsymbol{X}}_{t \mid t}, t=1,2, \ldots, T\right\}$. We then take the first components of these as our estimates $\left\{\hat{a}_{t}, t=1, \ldots, T\right\}$ of activation. The top panel in Figure 4 shows a subset of the time series of observations $y_{t}$ of pixel intensity (from the video frame in Figure 1), overlaid in red with the corresponding estimated activation $\hat{a}_{t}$. This subset comes from the first half of the third trial, encompassing the presentation of the 0 degree and 45 degree stimuli and the blank gray screen that follows each of these stimuli, and covers 240 frames or 8 seconds in total. The bottom panel 


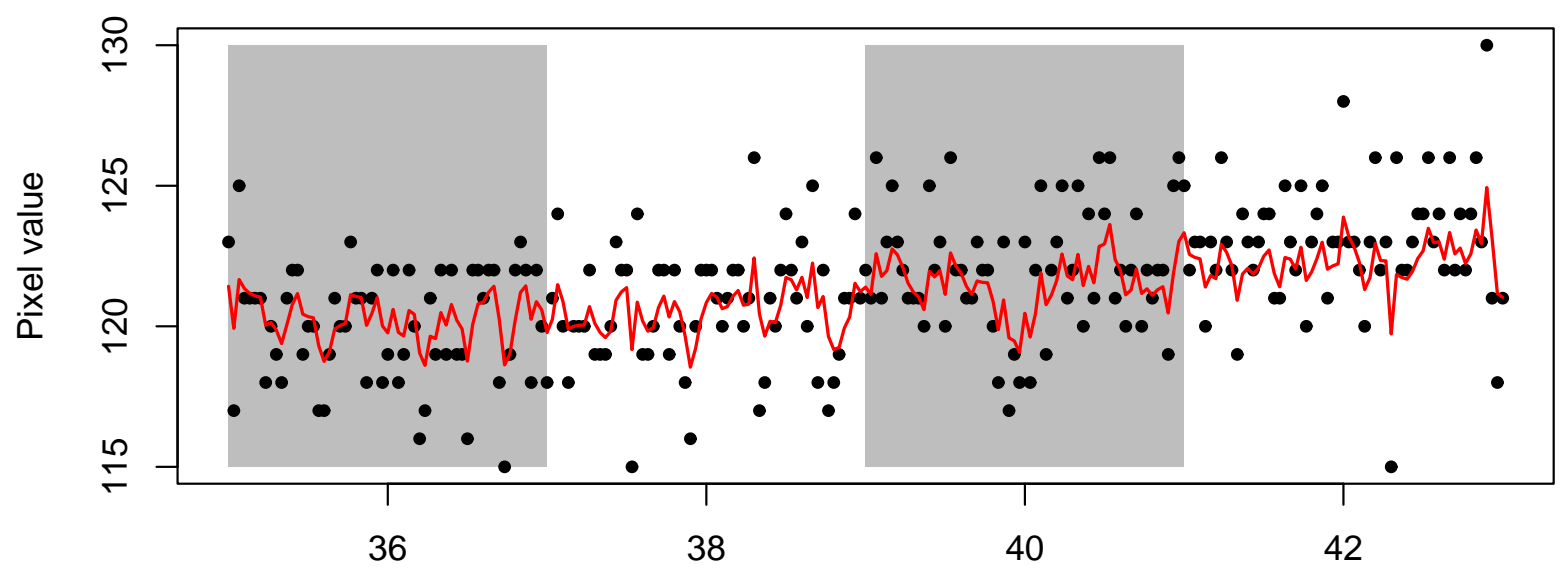

Time (seconds)

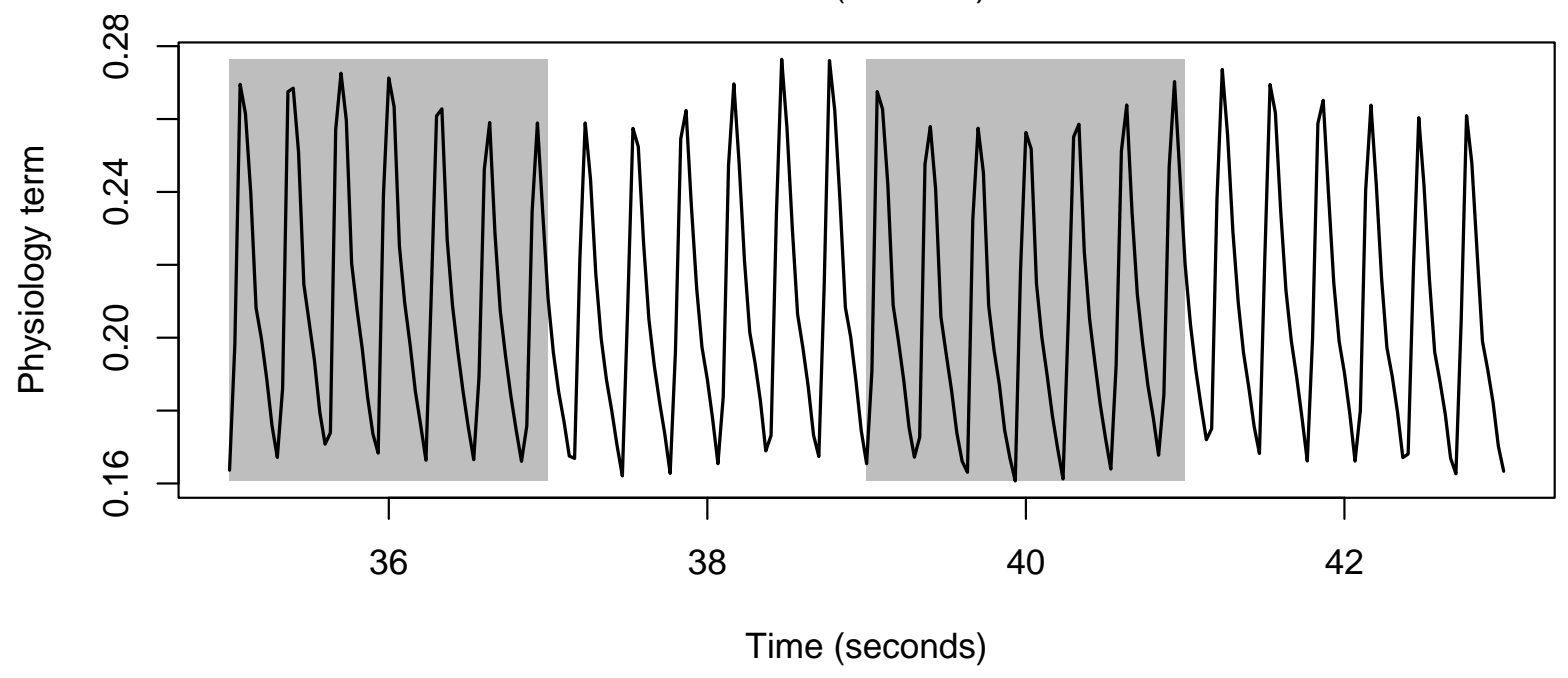

Figure 4: Top: Video data $y_{t}$ (black points) and estimates of activation $\hat{a}_{t}$ (red line) for a single pixel during the first half (first 240 frames) of the third trial. Bottom: The moving average of blood pressure defining our physiology $y_{t}$ term, shown over the same time period. Note the heartbeat and respiration components of this moving average; we want to remove these from our estimates of activation. In both plots the shaded regions indicate the onset and duration of the 0 degree stimulus (left) and the 45 degree stimulus (right) in this trial. 
of Figure 4 gives the moving average of blood pressure measurements during this same window of time, i.e., our physiology $t_{t}$ term from (14), using the estimated values $\hat{\beta}_{1}, \ldots, \hat{\beta}_{3}$.

The figure shows significant noise in estimated activation, making it difficult to identify stimulus-induced activation differences by eye. (Although there is an apparent increase in activation level over the 8 second window shown, this increase is not sustained over the entire time series.) This is consistent with the widely acknowledged low signal-to-noise ratios in optical imaging.

We can also perform residual analysis for our fitted model. To do this, we simply compute

$$
r_{t}=y_{t}-E\left(Y_{t} \mid Y_{t-1}=y_{t-1}, \ldots, Y_{1}=y_{1}\right)
$$

The conditional expectation is easily computed as $E\left(Y_{t} \mid Y_{t-1}=y_{t-1}, \ldots, Y_{1}=y_{1}\right)=G_{t} \hat{\boldsymbol{X}}_{t \mid t-1}$. Under the assumption of model "correctness" (i.e., that the data were generated by the model), these residuals should be realizations of independent identically distributed normal random variables with mean zero and some constant variance. The residuals for our pixel are shown in Figure 5, and clearly do not exhibit the strong periodic heartbeat- and respiration-related components in the original observations. They are also approximately normally distributed, as indicated by their histogram.

It is also interesting to consider the effect of each particular stimulus on activation at this pixel. Each of the $\gamma_{i}$ parameters determines a long-term activation level in the pixel for a given stimulus (the long-term activation level would be the stationary mean of the autoregressive process $\left\{a_{t}\right\}$ with stimulus $t$ held constant under a fixed stimulus). The fundamental premise of directional tuning in visual cortex is that localized regions exhibit high activity when the orientation of the visual stimulus is aligned with one particular "preferred" direction, and low activity when the orientation is perpendicular to the preferred direction. For this behaviour to be exhibited by our model, we would expect the $\gamma_{i}$ parameters (relative to the base level driving term $\gamma_{0}$ ) to exhibit the same directional preferences.

Figure 6 shows a plot of the values $\hat{\gamma}_{i}-\hat{\gamma}_{0}, i \in 1, \ldots, 4$, versus the corresponding angles $(0,45,90$, and 135 degrees) of stimuli, along with a fitted periodic (sinusoidal) curve. This sinusoid is maximized at a stimulus angle of 167 degrees, indicating that the preferred orientation for this pixel is just short of horizontal (180 degrees). This agrees with what we expected for this pixel based on examining standard difference maps (described in the introduction) for this experiment.

\section{Discussion}

We have demonstrated that a linear state-space model framework can be used to perform analysis of optical imaging data, and that, by appropriate specification of the model, it can be used to correct for artifacts induced by extraneous physiological processes. The framework provides the benefits of likelihood-based analysis of statistical models: one can check for goodness-of-fit and obtain optimal estimates under model "correctness" assumptions. Using the framework, it also becomes possible to perform hypothesis tests on parameters of interest by computing Hessian matrices to obtain confidence regions for our parameters. For instance, with the model we considered, one could construct a formal test statistic for the hypothesis that different stimuli lead to different pixel intensities.

It is also worth pointing out that Roweis and Ghahramani (Roweis and Ghahramani, 1999) have recast several traditional methods, like the principal component analysis and independent component analysis approaches used with optical imaging data, as special cases of linear state-space models. This makes it 


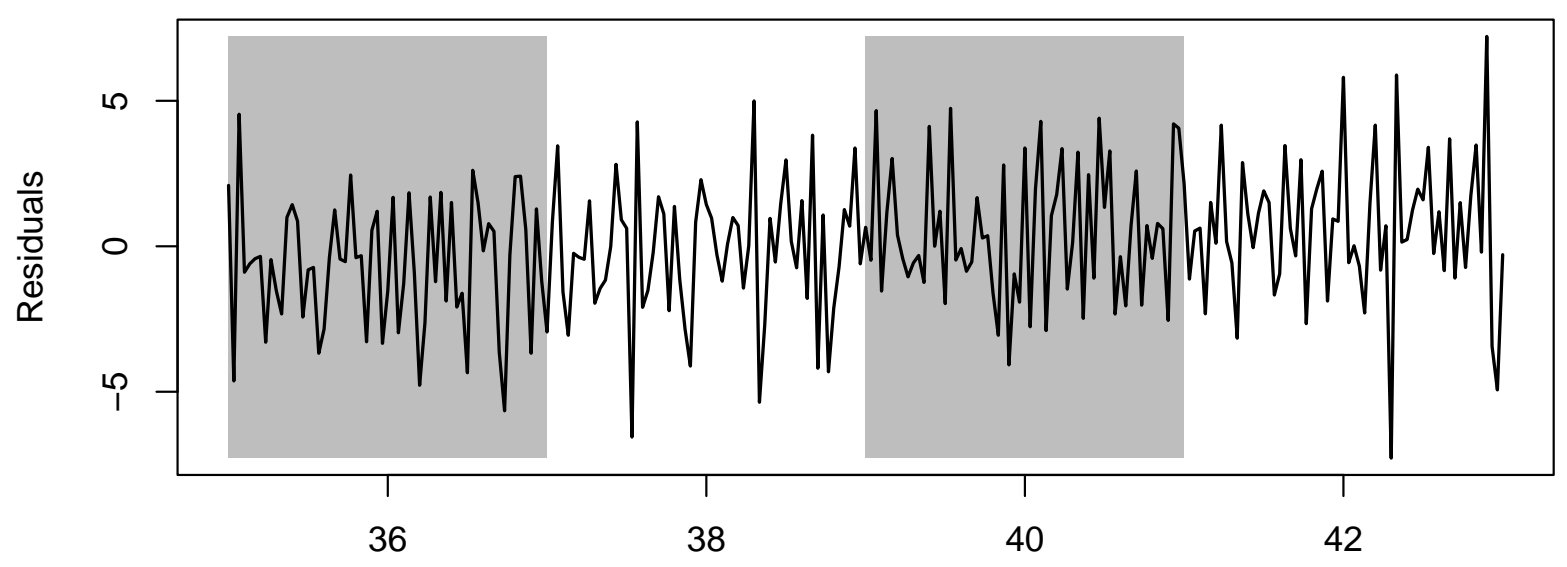

Time (seconds)

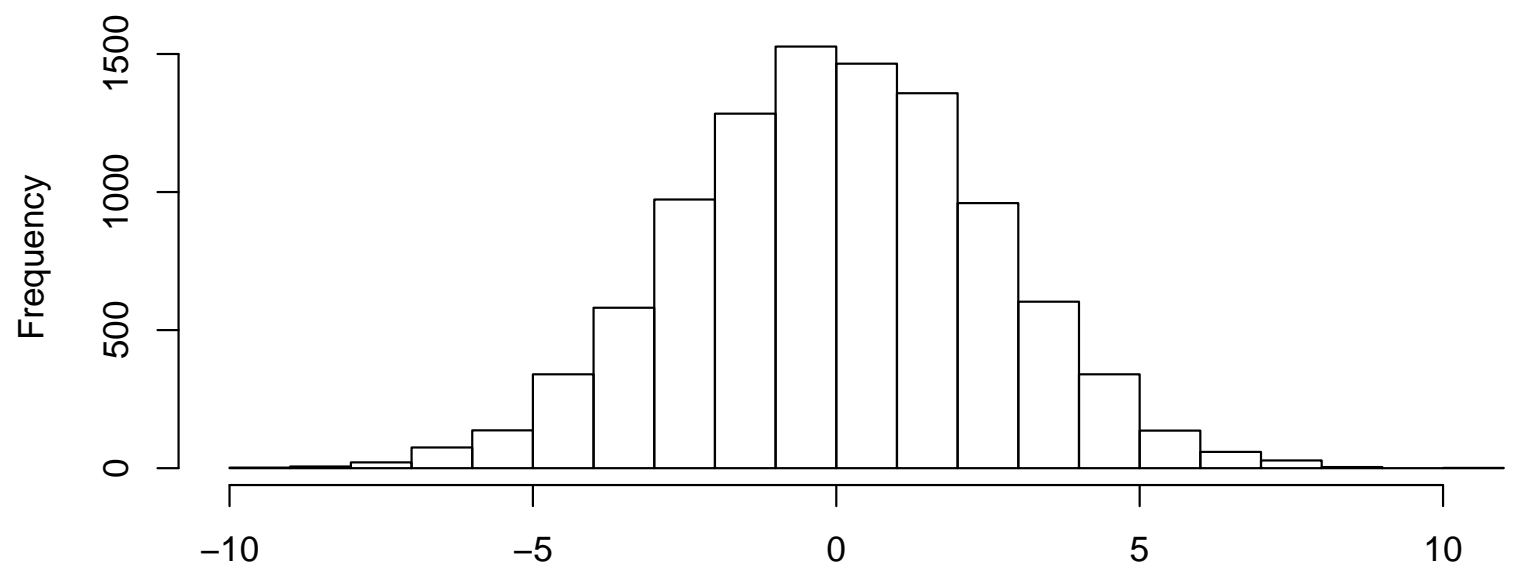

Residuals

Figure 5: Top: Residuals for the pixel and time period shown in Figure 4 as computed by $y_{t+1}-G_{t+1} \hat{\boldsymbol{X}}_{t+1 \mid t}$. These residuals show no evidence of heartbeat or respiration artifacts. Bottom: Histogram of the residuals, demonstrating normality. 


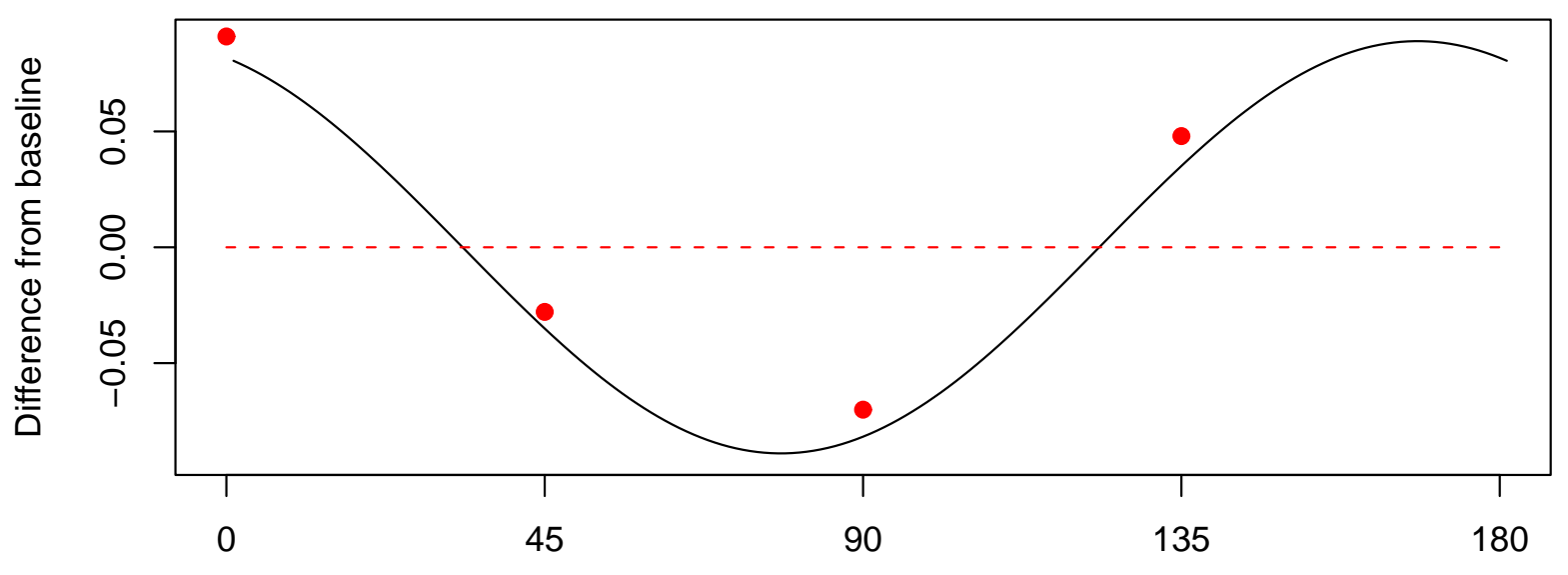

Angle of presented stimulus

Figure 6: The differences $\hat{\gamma}_{i}-\hat{\gamma}_{0}$ for each of the four stimuli, with corresponding stimulation angles on the $x$-axis. These quantities measure the instantaneous perturbation, relative to baseline level $\gamma_{0}$ induced in activation by each stimulus. The fitted sinusoid suggests that the preferred stimulus angle for this pixel is 167 degrees.

possible to perform direct comparisons between our models based on use of auxiliary measurements and current state of the art factoring methods for optical imaging.

In experiments to elicit orientation columns, the scientist typically knows what stimulus is being presented at each time $t$. It may also be of interest sometimes to estimate a stimulus based only on optical imaging data. (This would be a step toward developing methods to determine what the subject is "seeing", based only on analysis of brain activity.) In this case a filtering approach can also be used, by incorporating the (unobserved) stimulus into the state vector and developing an appropriate model for it. However, the state equation generally becomes nonlinear when this is done, making the Kalman filter no longer appropriate. In such cases, it would be natural to modify our approach by applying standard nonlinear and/or non-Gaussian filtering techniques ilke particle filtering methods (described in, e.g., (Kitagawa, 1996; Doucet et al., 2001; Gordon et al., 1993)).

There are several natural possibilities for extending and improving the framework described in this paper. One way would be simply to allow for nonlinear and/or non-Gaussian models for pixels, using the aforementioned particle filtering methods (or other nonlinear filtering approaches). Such models might fit the data better, and thus yield better filtered estimates of activation levels. In addition, one obvious feature in image data is the correlation structure between neighboring pixels. This could in theory be accounted for by creating a model with an enormous state vector $\boldsymbol{X}_{t}$ containing all pixel activation levels in the entire image. However, this would in general be computationally infeasible. Instead, it could be feasible to develop extensions of the framework that specify local correlation within spatial neighborhoods. We would expect such approaches to effectively decrease noise levels (relative to analyses based on individual pixel models), thereby improving signal resolution. 


\section{Acknowledgements}

We thank Seong-Gi Kim, Hiro Fukuda, and Chan Hong Moon for generously sharing their data and scientific expertise. Thanks also to Mike Hamada and Christine Anderson-Cook for their helpful feedback on an early draft of this paper. This work was supported in part by NIH Grants R21EB005967-01A1 and R01EB00584701 and by an AT\&T Labs Fellowship.

\section{References}

P.J. Brockwell and R.A. Davis. Time Series: Theory and Methods, Second Edition. Springer-Verlag New York, Inc., 1991.

A. Doucet, N. de Freitas, and N. Gordon, editors. Sequential Monte Carlo Methods in Practice. Springer, New York, 2001.

M. Gabbay, C. Brennan, E. Kaplan, and L. Sirovich. A principal components-based method for the detection of neuronal activity maps: Application to optical imaging. NeuroImage, 11:313-325, 2000.

N.J. Gordon, D.J. Salmond, and A.F.M. Smith. Novel approach to nonlinear/non-Gaussian Bayesian state estimation. IEE Proc. F, 140:107-113, 1993.

A. Grinvald, E. Lieke, R.D. Frostig, C.D. Gilbert, and T.N. Wiesel. Functional architecture of cortex revealed by optical imaging of intrinsic signals. Nature, 324(2):361-364, 1986.

A. Grinvald, D. Shoham, A. Shmuel, D. Glaser, I. Vanzetta, E. Shtoyerman, H. Slovin, C. Wijnbergen, R. Hildesheim, and A. Arieli. Modern Techniques in Neuroscience Research, chapter 34, pages 893-969. Springer Verlag, 1999.

A. Grinvald, H. Slovin, and I. Vanzetta. Non-invasive visualization of cortical columns by fMRI. Nature Neuroscience, 3(2):105-107, February 2000.

D. Hubel and T. Wiesel. Shape and arrangement of columns in cat's striate cortex. Journal of Physiology (London), 165:559-568, 1963.

M. Hübener and T. Bonhoeffer. The Cat Primary Visual Cortex, chapter 2, pages 131-166. Academic Press, 2002 .

R.E. Kalman. A new approach to linear filtering and prediction problems. Transactions of the ASMEJournal of Basic Engineering, pages 35-45, 1960.

G. Kitagawa. Monte Carlo filter and smoother for non-Gaussian nonlinear state space models. Journal of Computational and Graphical Statistics, 5(1):1-25, 1996.

K. Myers. The billion byte brain: Combining physiological data and gigabytes of images to improve maps of brain activity. In 2004 Proceedings of the American Statistical Association, 2004.

S. Ogawa, D. Tank, R. Menon, J.M. Ellermann, S.-G. Kim, K. Merkle, and K. Ugurbil. Intrinsic signal changes accompanying sensory stimulation: Functional brain mapping using MRI. In Proc. of the National Academy of Sciences, volume 89, pages 5951-5955, 1992. 
S. Roweis and Z. Ghahramani. A unifying review of linear gaussian models. Neural Computation, 11:305-345, 1999.

I. Schießl, M. Stetter, J. Mayhew, A. Askew, N. McLoughlin, J. Levitt, J. Lund, and K. Obermayer. Blind separation of spatial signal patterns from optical imaging records. In ICA99-International Workshop on Independent Component Analysis and Blind Source Separation, Aussois, France, pages 179-184, 1999.

M. Stetter, I. Schießl, T. Otto, F. Sengpiel, M. Hübener, T. Bonhoeffer, and K. Obermayer. Principal component analysis and blind separation of sources for optical imaging of intrinsic signals. NeuroImage, 11:482-490, 2000. 